\title{
Calibration of computer-monitored running wheels with adjustable drag
}

\author{
DOUGLAS WAHLSTEN, KATHERINE M. BISHOP, and ANNALEE KRUYER \\ University of Alberta, Edmonton, Alberta, Canada
}

\begin{abstract}
The design of a rodent running wheel with adjustable drag and ability to measure accurately the direction as well as the speed of running is described. A computer program in the $\mathrm{C}$ language enables a single microcomputer to monitor several wheels simultaneously. Drag is conveniently calibrated by the rate of deceleration from 1 revolution per second when no animal is present, and the calibration procedure can also assess the smoothness of the drag mechanism. Results of tests of the setability, repeatablility, and durability of the drag are presented. A method is outlined to determine the physical work required to accelerate the wheel and to maintain its rotation against the frictional drag.
\end{abstract}

Running wheels have long been a useful tool in examining a variety of physiological and psychological phenomena such as instrumental responses in learning (Brogden, Lipman, \& Culler, 1938; Iso, Brush, Fujii, \& Shimazaki, 1988), circadian rhythms (Golombek, Ortega, \& Cardinali, 1993; Morse et al., 1995; Peng \& Kang, 1984; Vitaterna et al., 1994), locomotor activity (Friedman, Garland, \& Dohm, 1992), the effects of intense exercise (Looy \& Eikelboom, 1989; Russell et al., 1989; Shyu, Andersson, \& Thorén, 1984), and the mode of inheritance of behaviors (Oliverio, Castellano, \& Messeri, 1972). Except for the studies of learning, the running wheels employed were usually simple apparatus that measured only the number of complete revolutions of the wheel. If the only measure of interest is number of complete rotations, many wheels can be observed with one computer. An apparatus designed by Petree, Haddad, and Berger (1992) provides more information and can be used with two wheels monitored simultaneously by the same computer (SzaldaPetree, Karkowski, Brooks, \& Haddad, 1994). The present report describes a computer-monitored running wheel apparatus that is capable of measuring finer details of running behavior, including time spent running, distributions of running speeds, number and duration of bouts, variance of running speeds within a bout, and direction

This research was supported in part by Grant OGP45825 from the Natural Sciences and Engineering Research Council of Canada and the Central Research Fund, University of Alberta. The work of K.B. and A.K. was supported by scholarships from NSERC. The authors are grateful to Dudley F. Peeler, Gunter Lorberg, Norman Standish, and Edward Thompson for assistance with the computer programming and to Lou Omerzu and Harold Tischer for construction of the apparatus. A. Kruyer is now at the Department of Psychology, University of Western Ontario. Requests for reprints or copies of computer programs should be addressed to D. Wahlsten, Department of Psychology, University of Alberta, Edmonton, AB, Canada T6G 2E9 (e-mail: wahlsten@psych.ualberta.ca). of running for an unlimited number of trials of any specified duration. In addition, the resistance or frictional drag of the wheel apparatus is adjustable and quantifiable, allowing for controlled testing of this factor for the first time. Twenty or more wheels can be used simultaneously with a reasonably fast microcomputer. Although the device reported here is intended for use with mice, the wheel itself could easily be enlarged for rats.

\section{Running Wheel Design}

Figure 1 shows the parts of the wheel apparatus. More detailed schematics are available from the first author (D.W.). A plastic disk attached to the shaft carries three small reflective strips that activate infrared photocells. Smooth, almost frictionless rotation can occur because the photocell transducer makes no direct contact with moving parts and the shaft is mounted on ball bearings. An adjustable drag mechanism on the shaft sets the impulse required to start rotation and the work needed to maintain it. We use a finely machined wheel with aluminum rims carrying stainless steel bars, but an inexpensive plastic wheel from a pet shop (Fritz Pet Products "play wheel") can also be attached to the hub with screws and requires less labor by a skilled machinist. Cost for the parts to build one apparatus with the plastic "play wheel" is about $\$ 50$, not including labor.

If there is only one reflective strip and photocell, the device cannot distinguish genuine running from simple back-and-forth swinging. Two strips and two photocells detect running as a movement that actuates photocell $\mathrm{B}$ only after photocell A has been crossed, or vice versa, whereas repeated actuation of the same photocell constitutes either swinging or a change of direction of running. However, two photocells cannot determine which direction of rotation is clockwise. Three photocells $(\mathrm{A}, \mathrm{B}, \mathrm{C})$, aligned on the same axis with reflective strips spaced $120^{\circ}$ 


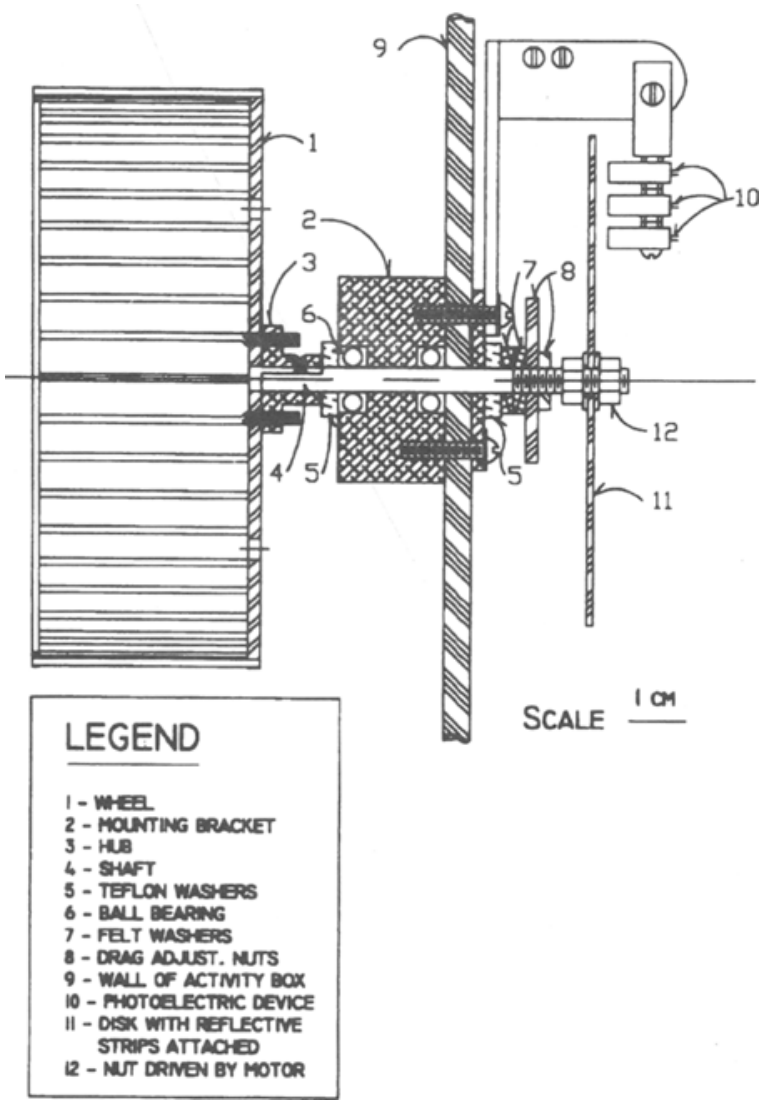

Figure 1. Side view of the running wheel proper (part 1), the ball-bearing mount (parts 2 and 6), the drag mechanism (parts 5,7 , and 8 ), the photocell-detection assembly (parts 10 and 11), and the drive nut (part 12) used during calibration of the drag. The entire apparatus is mounted on the wall of the plastic box (part 9) housing the animal. All parts are drawn to scale. Except for the bars affixed to the rims of the running wheel and the rims themselves (part 1), most of the other parts are shown in crosssection. Photocells to detect the reflective strips $A, B$, and $C$ in Figure 2a are indicated.

apart, can discriminate rocking from running and yield direction and speed of running (see Figure 2a). Size of the reflective strip must guarantee that the computer never misses the hit of a new strip when the mouse is running at top speed, which is greater than 1 revolution per second $(=60 \mathrm{rpm})$ but never more than 2 $(=120 \mathrm{rpm})$. If several wheels are monitored simultaneously, the strip must be larger because the computer is busier. With four wheels in operation, we have found that a strip covering $20^{\circ}$ of arc never was missed during the peak of mouse activity. When four wheels are turning at $120 \mathrm{rpm}$, one-quarter of a $20^{\circ}$ strip will be crossed in $7 \mathrm{msec}$, which is sufficient for several complicated operations on a $486 \mathrm{DX}-33$ computer.

The adjustable drag employs the same mechanism as the popular spinfishing reel. Washers, in this case made of Teflon and felt, are compressed by a thin nut on a threaded shaft (Figure 1), and the adjusting nut is locked in position by a second nut. Because of differences between de- vices and wear on the washers, the number of turns of the nut on the shaft is not a good indicator of drag. Instead, drag is calibrated by the rate at which a wheel decelerates when no mouse is present. For this purpose, an annulus (Figure $2 \mathrm{~b}$ ), made from 0.5 -mm aluminum, is employed to actuate the outermost photocell. The annulus has 24 equally spaced reflective strips, each covering $7.5^{\circ}$, generated with Corel Draw and printed on overhead transparency film at 1,200 dpi. The model annulus in our Figure $2 b$ could be enlarged with a photocopier and affixed to the disk. Calibration is done with a 60 -rpm precision synchronous motor (Princeton Industries Model PB) which has a long shaft with a socket at the end that fits loosely over the hexagonal nut on the wheel shaft (Figure 1 , item 12). When the socket is suddenly pulled away, the wheel begins to decelerate and the times between hits of strips gradually increase.

\section{Electronic Interface}

A reflective strip is detected by a Texas Instruments TIL139 infrared emitter-detector device that sends its output to an interface circuit that transmits a continuous $5-\mathrm{V}$ signal to the computer when the photocell is over a strip. This signal goes to an inexpensive digital input-output card built for us by our Department of Psychology shop for the IBM AT bus, although several commercially avail-

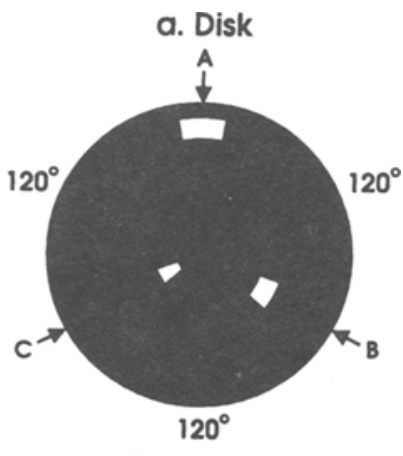

Scale $\underset{k \leftrightarrow 1}{2} \mathrm{~cm}$

b. Annulus

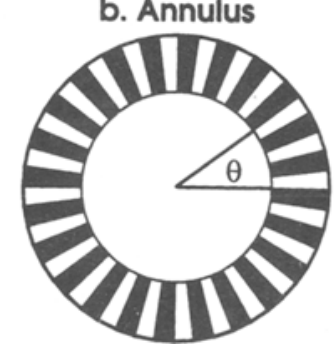

Figure 2. (a) Diagram of the disk, showing the locations of the three reflective strips that activate photocells $A, B$, and $C$, respectively. Each strip occupies $20^{\circ}$ of arc. (b) Diagram of the annulus, with 24 reflective strips, which is used to calibrate the drag mechanism. The angle from 0 of the leading edge of a particular strip as it reaches its photocell is indicated as $\theta$. The scale is shown for our device, although a smaller disk and annulus could be built. 
able digital I/O cards could also be adapted for this purpose. The card contains four chips (Intel 8255 PIA), each of which has three 8-bit parallel ports, and a timer chip (AMD 9513) that can be configured for 16- or 32-bit counting of a time base ranging from $1 \mathrm{MHz}$ to $100 \mathrm{~Hz}$. Thus, the state of each photocell is presented to the computer as 1 bit ( 0 or 1$)$ of an 8-bit byte that can be read from a port with the $\mathrm{C}$ language inportb() function. The registration delay for this interface setup is about $5 \mu \mathrm{sec}$ from when the photocell senses a strip to availability of this information in the central processing unit. In our experiments, the I/O card is employed with a $486 \mathrm{DX}-33$ computer to run four wheels simultaneously, but it can monitor as many as $96 / 3=32$ wheels if on-line computations are kept to a minimum. Because data are collected for several hours or days at a time, an uninterruptible power supply (American Power Conversion Smart-UPS 600) runs the computer and electronic interface during episodes of low or no line power. The digital I/O card currently costs $\$ 310$ and the electronic interface costs about $\$ 100$ per wheel for parts.

\section{Computer Programs}

All port address definitions, functions, and programs are written in the $\mathrm{C}$ language and compiled with Borland Turbo C. Five programs are used routinely to operate the wheels. TUNE.C sounds a $500-, 1000-$, or $1500-\mathrm{Hz}$ tone whenever photocell $\mathrm{A}, \mathrm{B}$, or $\mathrm{C}$, respectively, is crossed by a strip on any of the four wheels. This ensures that all photocells are properly aligned before an experiment is started. CALMOTOR.C calibrates the 60 -rpm motor, determining the mean and variance of time for one revolution. CALSTRIP.C determines the mean and variance of times between the three strips when the wheel is driven at $60 \mathrm{rpm}$ in either direction. This verifies the spacing of the strips. CALDRAG.C determines when the wheel has reached $60 \mathrm{rpm}$ and then computes the rate of deceleration, starting when time between two annulus strips (Figure $2 b$ ) first exceeds $0.0455 \mathrm{sec}$. The formula for computing deceleration is derived in the Appendix. 4wHEELS.C continuously monitors four wheels and collects a variety of measures of running and rocking behavior. The source code for all five programs is available from COMPsych under the file name WHEEL.ZIP. The file can be retrieved from the COMPsych BRMIC archive by anonymous ftp from gluon. hawk.plattsburgh.edu using Path $=$ pub/compsych/brmic and following general instructions provided by Hornby and Anderson (1994). Access will also be available over the World-Wide Web at http://www.plattsburgh.edu/ compsych. In addition, we will send copies via e-mail or regular mail.

\section{Calibration of Motor Speed and Strip Spacing}

Prior to utilizing the 60 -rpm motor, its speed was calibrated against the 32-bit clock using the outermost photocell. The motor was accurate to within about $0.1 \%$ for one revolution. When times for different strip pairs on a single wheel varied by more than $5 \%$ from $0.333 \mathrm{sec}$, the strip positions on the disk were adjusted. Error in the time between annulus strips (Figure $2 \mathrm{~b}$ ) was small (mean time $=0.0417 \mathrm{sec}, S D=1 \mathrm{msec}$ ). The measures of running speed obtained with this apparatus are considered to be within $5 \%$ of the true value, which is quite adequate for most purposes and is much less than the inherent variability from moment to moment in mouse behavior.

\section{Calibration of Drag}

Preliminary tests using Equation 5 in the Appendix revealed that the relationship between angular deceleration
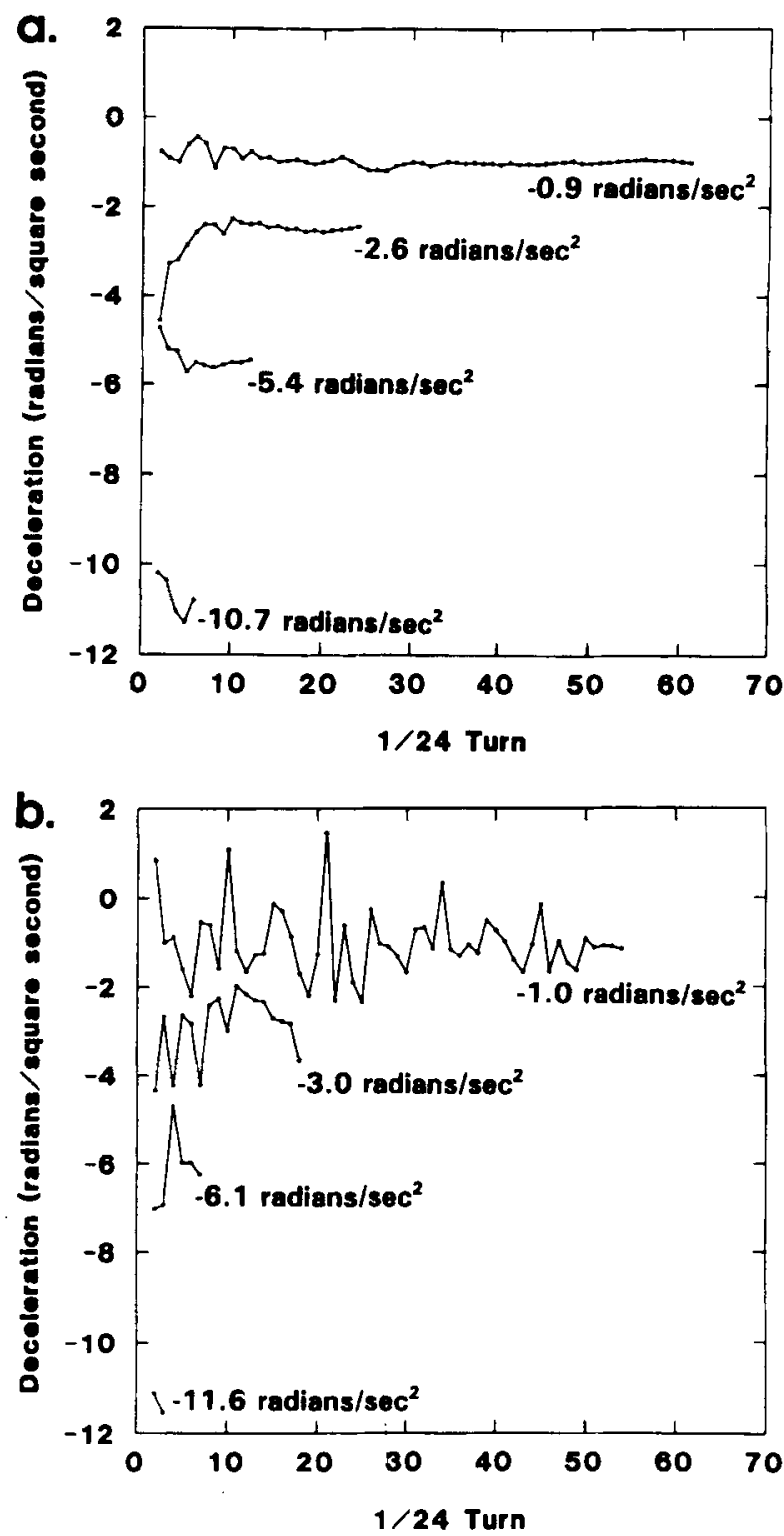

Figure 3. Measured deceleration ( $\alpha$ ) during each $1 / 24$ th of a turn of the annulus (Figure $2 b$ ) when the drag is set at different values. (a) The values are very stable, because the drag mechanism operates smoothly with new washers. (b) There is more fluctuation from moment to moment because the washers are worn after weeks of heavy use. In both cases, the deceleration is shown for all but the final $1 / 24$ th turn of the wheel. 
and number of 1/24th turns did not depart significantly from a straight line with slope of 0 , which implies that the rate of deceleration did not change systematically over several successive strips. However, deceleration $(\alpha)$ does sometimes vary substantially from moment to moment. Figure 3a shows results when smooth, unused washers were tested, whereas Figure $3 \mathrm{~b}$ reveals that after several weeks of use the washers were uneven and created a moderately fluctuating drag. For this reason, deceleration is computed by CALDRAG.C, using an average of all values up to but not including the interval when the wheel stops. Variance of deceleration provides a good estimate of smoothness of the drag mechanism and tells the operator when to replace worn washers. Drag settings possible when using this mechanism range from approximately -12 to $-0.2 \mathrm{rad} / \mathrm{sec}^{2}$. At the lowest possible value when the adjusting nut is backed off completely, $\alpha=-.02$ and our metal wheel requires about $30 \mathrm{sec}$ to stop in about 11.5 turns after starting at $60 \mathrm{rpm}$. The resistance of the drag was easily and accurately set. To demonstrate this, the number of trials to attain goal drag settings of $-10,-6,-3,-1.5$, and $-0.5 \mathrm{rad} / \mathrm{sec}^{2}$ were counted. In each case, it was found that the drag could usually be set within $25 \%$ of the goal in two trials and within $5 \%$ in five trials.

The short-term stability of deceleration was determined by obtaining 10 measurements at each of five drag levels $\left(-12,-6,-3,-1\right.$, and $\left.-0.5 \mathrm{rad} / \mathrm{sec}^{2}\right)$. There were no significant differences across trials for any of the five drag levels (all $p s>.10$ ). The reliability of the deceleration measurement procedure was tested by taking three consecutive measurements at each of 24 drag levels over the full range of resistances. The correlations between measurements were $r=.96$ between Trials 1 and $2, r=$ .97 between Trials 2 and 3 , and $r=.94$ between Trials 1 and 3 . Clockwise and counterclockwise decelerations were measured at 12 drag levels over the range of possible resistance settings, and the correlation between the clockwise and counterclockwise measurements was very high $(r=.990)$. Because the washers used to control the resistance of the wheel may loosen or tighten over a period of running, the change in drag over $12 \mathrm{~h}$ of intense running was determined. Ten estimates of deceleration were obtained before and 10 were obtained after an overnight period of running by a test mouse. Five drag settings $\left(-12,-6,-3,-1\right.$, and $\left.-0.5 \mathrm{rad} / \mathrm{sec}^{2}\right)$ were tested in separate cases. No significant change was detected for drag levels of -0.5 and $-12(t$ tests, $p s>.10)$, but there was a change in drag levels of $-6,-3$, and $-1(p s<.001)$. However, there was no consistent direction of change in the drag; it increased for the -6 and -3 settings and decreased for the -1 setting. In all further tests, the drag level of the wheel was defined as an average of two measurements taken before the running period and two taken afterwards. Changes in the drag level over a prolonged period of testing make it important to recalibrate the drag. We routinely do this each day during the light phase of the activity cycle when mice are inactive.

\section{Mice on the Wheel}

Most mice run voluntarily and with great vigor on these wheels, including one which ran for $6.3 \mathrm{~h}$ in 1 day, completing 27,574 revolutions, or a linear distance of $12.2 \mathrm{~km}$, at a drag setting of $-3 \mathrm{rad} / \mathrm{sec}^{2}$, thereby expending 176 joules, or about 42 calories of energy, in overcoming the drag (see Appendix). A frequency distribution of rotational velocity for one-third turns of the wheel for a single mouse in 1 day revealed no clear boundary between running and nonrunning. Log-survivorship plots (see Slater \& Lester, 1982) yielded a criterion of $.45 \mathrm{sec}$ that did not correspond very well with direct observations. Video-taped sequences revealed that whenever the time between successive hits of two strips exceeded $75 \mathrm{sec}$, the mouse had indeed stopped running, at least momentarily. Using this definition, our program currently determines the number of complete rotations and swings (crossings of the same photocell), total time spent running, distance run, average running speed, maximum speed, modal speed, variance of speed within a bout, number of bouts, as well as frequency distributions of speed for each onethird turn and bout length. Data can be collected for virtually any desired time period, and results can also be recorded for successive blocks of time. Simple modifications to the program allow measurement of many other aspects of running, including direction of running.

\section{Discussion}

It is desirable that a running wheel involve enough frictional drag for the wheel to cease rotation soon after the animal leaves it. At the same time, if the drag is too strong, considerable energy will be expended to keep the wheel turning when the animal is running. A drag sufficient to stop the wheel in one-quarter to one-half turn would probably be acceptable for most purposes. This requires a setting of about $-6 \mathrm{rad} / \mathrm{sec}^{2}$ (see Figure 3 ). The ability to set the drag at such a level each day of an experiment should allow greater comparability of results across laboratories and between wheels and days in the same experiment. Our method and the equations presented in the Appendix could also be used with fixed-drag systems such as that of Szalda-Petree et al., (1994) to calibrate different wheel configurations and determine the energy required to overcome drag in any apparatus.

If the main focus of investigation is the total amount of running over a long period of time, the smoothness of the drag is not likely to be an important factor, because inertia of the spinning wheel tends to keep it moving over rough spots and the average decelerative drag through one revolution or an average over several tests with faster stopping can be used to calculate energy expenditure. However, if the finer details of running, especially during the early phase of learning to use the wheel, are of concern, the ability to measure and adjust smoothness of the drag mechanism may provide better data.

Several improvements over the present design can be anticipated. Perhaps a better drag mechanism with more disks of more durable material and compressed by a spring 
will maintain a smoother resistance. It would also be helpful to use four photocells per wheel and an integral calibration annulus to activate the fourth photocell, thereby eliminating the need to attach the annulus each day.

Using a drag of known deceleration will allow some interesting evaluations of the energetics of running. Wheel running raises body temperature (Golombek et al., 1993) and maximal oxygen consumption (Friedman et al., 1992), whereas running can temporarily reduce eating (Bauman, 1992), and food restriction can induce intense running (Pierce \& Epling, 1994). The physiology and psychology of this behavior are obviously complex. The temporal pattern of running can be strongly modulated by the availability of milk reinforcement during short daily sessions, even when the reward is not contingent on running (White, 1985). The force required to initiate wheel turning can influence the rate of task acquisition and resistance to extinction (Haddad, Szalda-Petree, Karkowski, Foss, \& Berger, 1994; Mowrer \& Jones, 1943), but many factors influence the results; these include large differences between individuals (Haddad et al., 1994; White, 1985) and the properties of the schedule of reinforcement (Haddad et al., 1994). Deliberately varying the precise amount of work required to turn the wheel using our calibration procedure may aid comparisons between laboratories and provide additional insights into the regulation of wheel running.

\section{REFERENCES}

Bauman, R. A. (1992). The effects of wheel running, a light/dark cycle, and the instrumental cost of food on the intake of food in a closed economy. Physiology \& Behavior, 52, 1077-1083.

Brogden, W. J., Lipman, E. A., \& Culler, E. (1938). The role of incentive in conditioning and extinction. American Journal of Psychology, 51, 109-117.

Friedman, W. A., Garland, T., \& Dohm, M. R. (1992). Individual variation in locomotor behavior and maximal oxygen consumption in mice. Physiology \& Behavior, 52, 97-104.

GolombeK, D. A., Ortega, G., \& Cardinali, D. P. (1993). Wheel running raises body temperature and changes the daily cycle in golden hamsters. Physiology \& Behavior, 53, 1049-1054.

Haddad, N. F., Szalda-Petree, A., Karkowski, A., Foss, R. L., \& BERGER, L. H. (1994). Wheel-running in discrete trial and operant paradigms under various effort requirements. Physiology \& Behavior, 56, 487-493.

HORNBY, P. A., \& ANDERSON, M. D. (1994). COMPsych: The electronic software information service and PC software archive for psychology. Behavior Research Methods, Instruments, \& Computers, 26, 57-59.

Iso, H., Brush, F. R., Fujil, M., \& Shimazaki, M. (1988). Runningwheel avoidance learning in rats (Rattus norvegicus): Effects of contingencies and comparisons of different strains. Journal of Comparative Psychology, 102, 350-371.

LoOy, H., \& EiKELBoom, R. (1989). Wheel running, food intake, and body weight in male rats. Physiology \& Behavior, 45, 403-405.

Morse, A. D., Russell, J. C., Hunt, T. W. M., Wood, G. O., Epling, W. F., \& PIERCE, W. D. (1995). Diurnal variation of intensive running in food-deprived rats. Canadian Journal of Physiology \& Pharmacology, 73, 1519-1523.

MOWRER, O. H., \& JONES, H. M. (1943). Extinction and behavior variability as functions of effortfulness of task. Journal of Experimental Psychology, 33, 369-386.

Oliverio, A., Castellano, C., \& Messeri, P. (1972). Genetic analysis of avoidance, maze, and wheel-running behaviors in the mouse. Journal of Comparative \& Phvsiological Psychology, 79, 459-473.

PENG, M. T., \& KANG, M. (1984). Circadian rhythms and patterns of running-wheel activity, feeding and drinking behaviors of old male rats. Physiology \& Behavior, 33, 615-620.

Petree, A. D., Haddad, N. F., \& Berger, L. H. (1992) A simple and sensitive method for monitoring running-wheel movement. Behavior Research Methods, Instruments, \& Computers, 24, 412-413.

Pierce, W. D., \& Epling, W. F. (1994). Activity anorexia: An interplay between basic and applied behavior analysis. Behavior Analyst, 17, 7 23.

Russell, J. C., Amy, R. M., Manickavel, V., Dolphin, P. J., Epling, W. F., PIERCE, D., \& BOER, D. P. (1989). Prevention of myocardial disease in JCR:LA-corpulent rats by running. Journal of Applied Physiology, 66, 1649-1655.

Shyu, C., Andersson, S. A., \& Thorén, P. (1984). Spontaneous running in wheels. A microprocessor assisted method for measuring physiological parameters during exercise in rodents. Acta Physiologica Scandinavica, 121, 103-109.

Slater, P. J. B., \& LeSTER, N. P. (1982). Minimising errors in splitting behaviour into bouts. Behaviour, 79, 153-161.

Szalda-Petree, A. D., Karkowski, A. M., Brooks, L. R., \& HadDad, N. F. (1994). Monitoring running-wheel movement using a serial mouse and ar IBM-compatible system. Behavior Research Methods, Instruments, \& Computers, 26, 54-56.

Vitaterna, M. H., King, D. P., Chang, A.-M., Kornhauser, J. M., Lowrey, P. L., McDonald, J. D., Dove, W. F., Pinto, L. H., Turek, F. W., \& TAKAHASKI, J. S. (1994). Mutatgenesis and mapping of a mouse gene, Clock, essential for circadian behavior. Science, 264, 719-725.

WhITE, J. M. (1985). Schedule-induced wheel-running: Effects of exposure to the schedule. Physiology \& Behavior, 34, 119-122.

\section{APPENDIX \\ Physics of the Running Wheel}

\section{Computation of Drag}

For derivation of rotational deceleration, the following symbols are used (see Figure 2b):

$\theta_{k}=$ angle of leading edge of strip $k$ from 0 in radians,

$\Delta \theta=$ angle between successive, equally spaced strips,

$\omega_{k}=$ rotational velocity in radians/second when strip $k$ is hit,

$\alpha_{k}=$ rotational acceleration $(d \omega / d t)$ in radians $/$ second $^{2}$ during the interval from strip $k-1$ to $k$.

$t_{k}=$ time when strip $k$ is hit, calculated from start of the test, $\Delta t_{k}=$ time to go from strip $k-1$ to strip $k$.

Provided the calibration annulus has many strips ( 24 in this case), it is reasonable to treat acceleration during the interval between successive strips as constant. In fact, actual measures indicate that $\alpha$ does not tend to change systematically over several successive strips.

When strip $k$ is reached after strip $k-1$ has been traversed, velocity is

$$
\omega_{k}=\omega_{k-1}+\alpha_{k} \Delta t_{k}
$$

and angular displacement is

$$
\theta_{k}=\theta_{k-1}+\omega_{k-1} \Delta t_{k}+\frac{\alpha_{k}}{2}\left(\Delta t_{k}\right)^{2} .
$$

Rearranging and substituting Equation 1 into Equation 2 yields

$$
\omega_{k}=\frac{\Delta \theta}{\Delta t_{k}}+\frac{\alpha_{k}}{2} \Delta t_{k} .
$$

Likewise, at the start of strip $k+1$,

$$
\Delta \theta=\omega_{k} \Delta t_{k+1}+\frac{\alpha_{k+1}}{2}\left(\Delta t_{k+1}\right)^{2} .
$$

Assuming that $\alpha \approx \alpha_{k} \approx \alpha_{k+1}$ over a small interval of $2 \Delta \theta$, and substituting Equation 3 into Equation 4 , 


$$
\alpha=\frac{2 \Delta \theta\left(\frac{1}{\Delta t_{k+1}}-\frac{1}{\Delta t_{k}}\right)}{\Delta t_{k}+\Delta t_{k+1}} .
$$

When deceleration results in $\Delta t_{k+1}>\Delta t_{k}, \alpha$ is negative.

\section{Rotational Energy and Torque}

The kinetic energy $\left(E_{\mathrm{k}}\right)$ of the moving wheel depends on its moment of inertia $(I)$ according to the relation $E_{\mathrm{k}}=(1 / 2) I \omega^{2}$. Moment of inertia is computed from the mass (mass $=$ volume $X$ density) of each component, its shape, and its distance from the axis of the shaft. Mass of the wheel proper (component 1 in Figure 1) is $77.5 \mathrm{~g}$, and that of the shaft, washers, disk, and nuts is $68.2 \mathrm{~g}$. The moment of inertia of the wheel proper $\left(I=3,218.7 \mathrm{~g} \mathrm{~cm}^{2}\right)$, most of which $\left(2,970.8 \mathrm{~g} \mathrm{~cm}^{2}\right)$ is attributable to the rods and rims furthest from the center, is far greater than that of the shaft, washers, disk, and nuts $\left(I=438.2 \mathrm{~g} \mathrm{~cm}^{2}\right)$ nearer the axis of the wheel. Thus, any substantial reduction in the moment of inertia of the moving parts must be achieved by using lighter plastic or graphite for the outer rims and rods. For example, a plastic running wheel (Fritz Pet Products "play wheel for small animals") from a local pet store weighs $22 \mathrm{~g}$ and has a much smaller moment of inertia $\left(1,158 \mathrm{~g} \mathrm{~cm}^{2}\right)$, although it is also less durable than a metal wheel.
Suppose the wheel is at rest and then the mouse accelerates it smoothly to velocity $\omega$ in $t \mathrm{sec}$. Because of the drag mechanism, the mouse does two kinds of work, one to accelerate the frictionless wheel and the other to overcome frictional drag. The total torque $(\Gamma)$ it must exert on the wheel is therefore a sum of accelerative (a) and drag (d) components, such that $\Gamma=\Gamma_{a}+\Gamma_{d}$, and the work done to move it through an angle $\theta$ to reach velocity $\omega$ when $\Gamma$ is constant is $W=W_{a}+W_{d}$. The accelerative portion is $W_{a}=(1 / 2) I \omega^{2}$. The torque exerted by the drag is $\Gamma_{d}=$ $I \alpha_{d}$ and the work done against the drag while the wheel accelerates smoothly to $\omega$ is $W_{d}=I \alpha_{d} \theta=(1 / 2) I \alpha_{d} \omega t$. To accelerate our metal wheel to $60 \mathrm{rpm}(2 \pi \mathrm{rad} / \mathrm{sec})$ in $1 \mathrm{sec}$ requires $W_{a}=$ 72,113 ergs of energy. When drag is set at $-5 \mathrm{rad} / \mathrm{sec}^{2}, W_{d}=$ 57,444 ergs. Once the wheel is turning smoothly at velocity $\omega=2 \pi \mathrm{rad} / \mathrm{sec}$, the work required to overcome the drag for $60 \mathrm{sec}$ is $W_{d}=I \alpha_{d} \omega t=6,893,283$ ergs, or about 0.69 joules, which is equivalent to 0.16 calories. Thus, the work required to accelerate the wheel to a typical running speed is similar to the work required to overcome drag during acceleration, whereas the work to maintain a steady speed for a minute greatly exceeds the quantity needed to accelerate the wheel.

(Manuscript received July 28, 1995; revision accepted for publication January $18,1996$. 